\title{
THE STRUCTURE OF INCOMPLETE DESTRUCTIVE BREAKDOWN SPOTS IN THIN POLYMER FILMS
}

\author{
S. SAPIEHA, W. JABLONSKI and M. KRYSZEWSKI \\ Centre of Molecular and Macromolecular Studies, Polish Academy of Sciences, Lódź 40, Poland
}

(Received June 1, 1973; in final form November 10, 1973)

\begin{abstract}
Experimental observations are given on the structure of breakdown spots in thin layers of polystyrene and polybenzene obtained by a glow discharge technique. The levels of energy associated with different types of breakdown are identified and it is shown that within the range of breakdown energy level, lower than $10^{-5}$ joules, non-destructive breakdown takes place. At higher energies, partial destruction of the breakdown channel occurs and with energy levels greater than $5 \times 10^{-3}$ joules, single hole, self-healing breakdown with complete destruction of the inner morphological channel structure occurs.
\end{abstract}

The nature of the breakdown is discussed in electrical and electronmicroscopy terms.

\section{INTRODUCTION}

The problem of channel structure in self-healing breakdown in thin polymer films is more interesting than in the case of simple inorganic compounds ${ }^{1,2,3}$. This is because of the more complex thermal degradation mechanism of a macromolecular dieletric and because of the formation of supermolecular structures in thin polymer films. In these thin layers exists the possibility of forming a more differentiated physical structure of breakdown channels.

Depending on experimental conditions the following types of breakdown event may take place:

a) destructive, causing total destruction of the specimen,

b) propagating self-healing, destroying large areas of the electrode and dielectric material, without, however, destroying the sample as whole,

c) single hole self-healing, eliminating weak spots,

d) nondestructive, bringing about local changes in the crystallinity of the sample if anything.

The prerequisite of self-healing breakdown is small thickness of the electrode, contained usually within the range of $100-2000 \AA^{1,2,3}$. Power density produced in the breakdown channel reaches the value of $10^{9} \mathrm{~W} / \mathrm{cm}^{2}$ and can only be compared with the power of giant-pulse lasers. In the case of simple inorganic compounds, the dielectric and electrode material evaporates in the course of the breakdown event, which has been confirmed in the spectrum investigations of the light emitted by the explosion products. The temperature in the breakdown channel ascertained on the basis of spectrum line intensity analysis was estimated to equal $4000-4500^{\circ} \mathrm{K}^{1,2}$.

In the present study, attention has been focussed on the formation of the physical structure of the breakdown channel in single hole self-healing breakdowns with partial destruction of the interior of the breakdown channel. The choice of material for investigation, thin polybenzene and polystyrene films, was governed by two factors: (a) the supermolecular structure of these films was already fairly well known ${ }^{5,6,7}$, and (b) the structure forming in the conducting breakdown channel could be compared with the surface structure of these films.

\section{EXPERIMENTAL}

Thin polystyrene and polybenzene films were obtained using the glow discharge method in the vapours of appropriate monomers. The thickness of the films under examination was within the range of 930-3000 \&. The films were provided with gold electrodes evaporated under vacuum $10^{-5} \mathrm{Tr}$. The upper electrode was $900-4000 \AA$ thick guaranteeing the formation of single hole self-healing breakdowns. The thickness of the films and electrodes obtained was determined by the multibeam interference method.

The scheme of the electrical system employed to obtain self-healing breakdowns is shown in Figure 1. The basic elements of the circuit are: high-ohmic resistor $R_{1}$ and capacity $C_{1}$ and sample capacity $C_{S}$ 


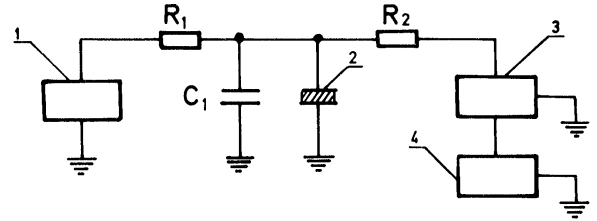

FIGURE 1 The scheme of the electrical system employed to obtain breakdowns

1 - voltage source, 2 - sample, 3 - electrometer Vakutronic VAJ 51, 4 - x-y recorder Endim 220 0/1, $R_{1}, R_{2}$-high-ohmic resistor, $C_{1}$-parallel capacity.

ensuring the time constant of the system of the order of a few seconds, and strictly controlled breakdown energy ${ }^{8}$. The breakdown event in the film took place on the ascending slope of the voltage curve at voltage $U_{b}$ and manifested itself in a sharp voltage drop down to some $U_{\min }$ value. Breakdown energy was calculated from the relation:

$$
\mathrm{E}=\frac{\mathrm{C}_{1}+\mathrm{C}_{\mathrm{s}}}{2}\left(\mathrm{U}_{\mathrm{b}}^{2}-\mathrm{U}_{\min }^{2}\right)
$$

The value of $E$ could be modified by varying the capacity $C_{1}$. The value of breakdown voltage $U_{b}$ and the value of minimal voltage $U_{\text {min }}$ were read from the diagram $U=f(t){ }^{8}$

In order to obtain the incompletely destroyed single hole breakdown, electrostatic discharge energy in the range $1.10^{-5}-5.10^{-3} \mathrm{~J}$ was used. The structures of the breakdown channels were examined using optical and electron microscopes. Samples to be examined by electron microscopy were obtained using the carbon replicas technique in three stages:

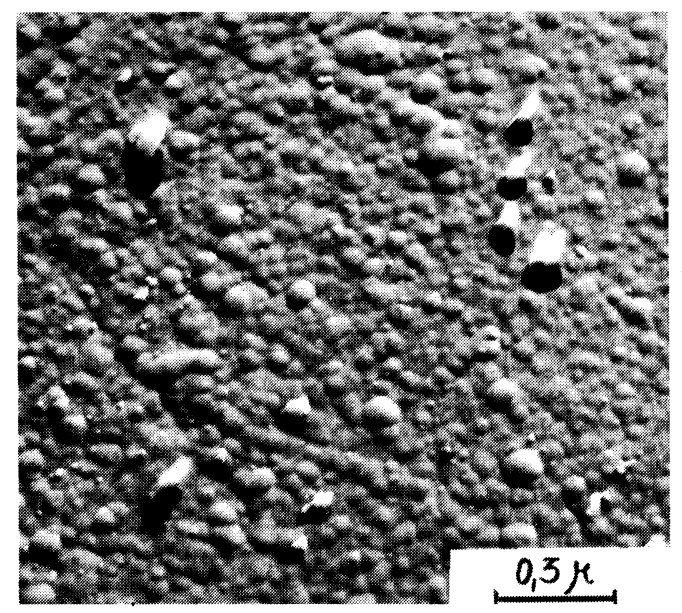

FIGURE 2 Surface structure of polybenzene film $5000 \AA$ thick. (a) evaporating of a thin carbon layer of thickness $100 \AA$, (b) contrasting with palladium and platinium under the angle of $30^{\circ}$, (c) evaporating of a suitable carbon replica of thickness up to $1000 \AA$. The replicas were then removed by means of polyacrylic acid.

Thin polystyrene and polybenzene films are characterized by a well developed surface structure $^{5,6}$. A typical electron micrograph of a polybenzene film surface $5000 \AA$ thick is demonstrated in Figure 2. In thicker polybenzene films, above $5000 \AA$, spherulite structures were also found. The size of the spherulites increases with the thickness of the film. For a film $4 \mu \mathrm{m}$ thick spherulite diameter is $30 \mu \mathrm{m}$. Figure 3 demonstrates that spherulites arise particularly readily along the cracks in the support which is testified by their great surface density.

\section{RESULTS}

The energy $E$ of electric discharge is expended on electrode and polymer film evaporation. The physical process of metallic electrode evaporation is well known and an energetic balance for such a case can be made easily. Macromolecular dielectrics, however, undergo destruction in high temperature either through burning or pyrolysis. The full picture of the destruction mechanism can be obtained when all the transitional stages of thermal destruction and all the products arising in these reactions are known.

In the present study the following stages of polymer film destruction were considered:

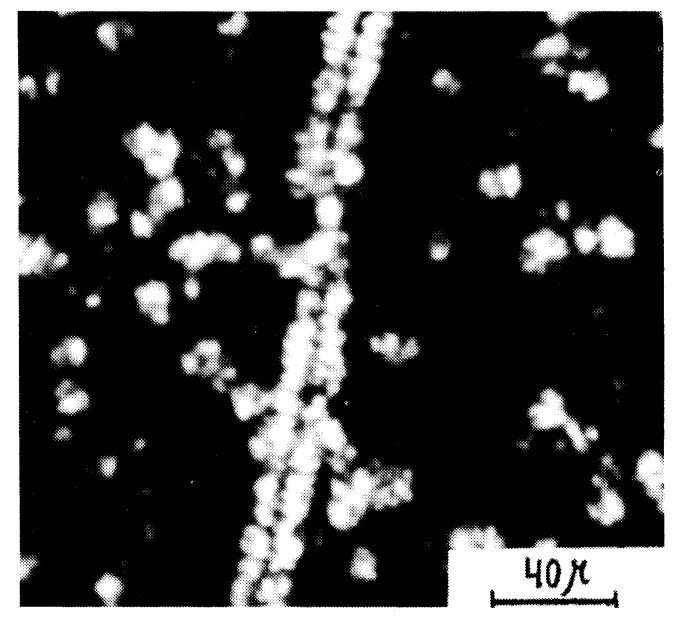

FIGURE 3 Spherulite formation in polybenzene film along the cracks in the support. 
TABLE I

Energetics of single hole breakdown for polystyrene

\begin{tabular}{|c|c|c|c|c|c|c|c|c|c|}
\hline$E\left(10^{4} \mathrm{~J}\right)$ & $E\left(10^{4} \mathrm{cal}\right)$ & $S_{m}\left(10^{5} \mathrm{~cm}^{2}\right)$ & $S_{p}\left(10^{5} \mathrm{~cm}^{2}\right)$ & $m_{m}\left(10^{9} \mathrm{~g}\right)$ & $m_{p}\left(10^{9} \mathrm{~g}\right)$ & $Q_{i m}\left(10^{7} \mathrm{cal}\right)$ & $Q_{i p(}\left(10^{7} \mathrm{cal}\right)$ & $k(\%)$ & $\eta(\%)$ \\
\hline 4.5 & 1.1 & 2.6 & 2.2 & 6.0 & 0.6 & 38.6 & 4.1 & 10.6 & 3.9 \\
\hline 9.0 & 2.2 & 4.8 & 4.0 & 11.1 & 1.1 & 71.2 & 7.4 & 10.4 & 3.6 \\
\hline 13.5 & 3.2 & 8.1 & 7.1 & 18.8 & 1.9 & 120.2 & 13.1 & 10.9 & 4.2 \\
\hline 25.0 & 6.2 & 12.0 & 9.6 & 27.8 & 2.6 & 173.0 & 17.7 & 10.2 & 3.1 \\
\hline 46.8 & 12.3 & 19.5 & 14.8 & 45.2 & 4.0 & 289.4 & 27.2 & 9.4 & 2.6 \\
\hline
\end{tabular}

a) a flux of Joule heat penetrating into the polymer,

b) heating of polymer to melting temperature $T_{\mathrm{m}}$,

c) dissociation connected with decomposition of the polymer into monomer and other final products as well as bond dissociation,

d) evaporation of degradation products.

A definite amount of heat is necessary for each of these processes and the heat required to bring about polymer destruction will be the total needed for the successive stages of the transition. The heat required for the evaporation of polystyrene degradation products was calculated for the degradation components/pyrolysis temperature $-1125^{\circ} \mathrm{C}$, styrene $63.7 \%$, toluene and ethylobenzene $5.8 \%$, benzene $13 \%$ given in Ref. 4 . Total heat $\Sigma Q_{i p}$ required for the evaporation of the mass $m_{p}$ and the surface $S_{p}$ of the polymer is given in Table 1 . The same Table gives the calculated heat needed for the evaporation of the electrode of mass $m_{m}$ and the surface $S_{m}$. It appears from the Table that although all the main processes of heat consumption by the polymer have been taken into account the total is considerably less than the heat necessary for the evaporation of electrodes $\Sigma Q_{i m}$ and equals $\mathrm{k} \cdot \Sigma Q_{i m}$ where the values $k$ are given in the ninth column of the Table for different breakdown energy values. Due to this, when drawing up the rough heat balance, the heat $\Sigma Q_{i p}$ spent on the evaporation of the polymer may be ignored and the resultant error will not exceed $12 \%$.

Calculations quoted above are in good agreement with theoretical considerations concerning energetic
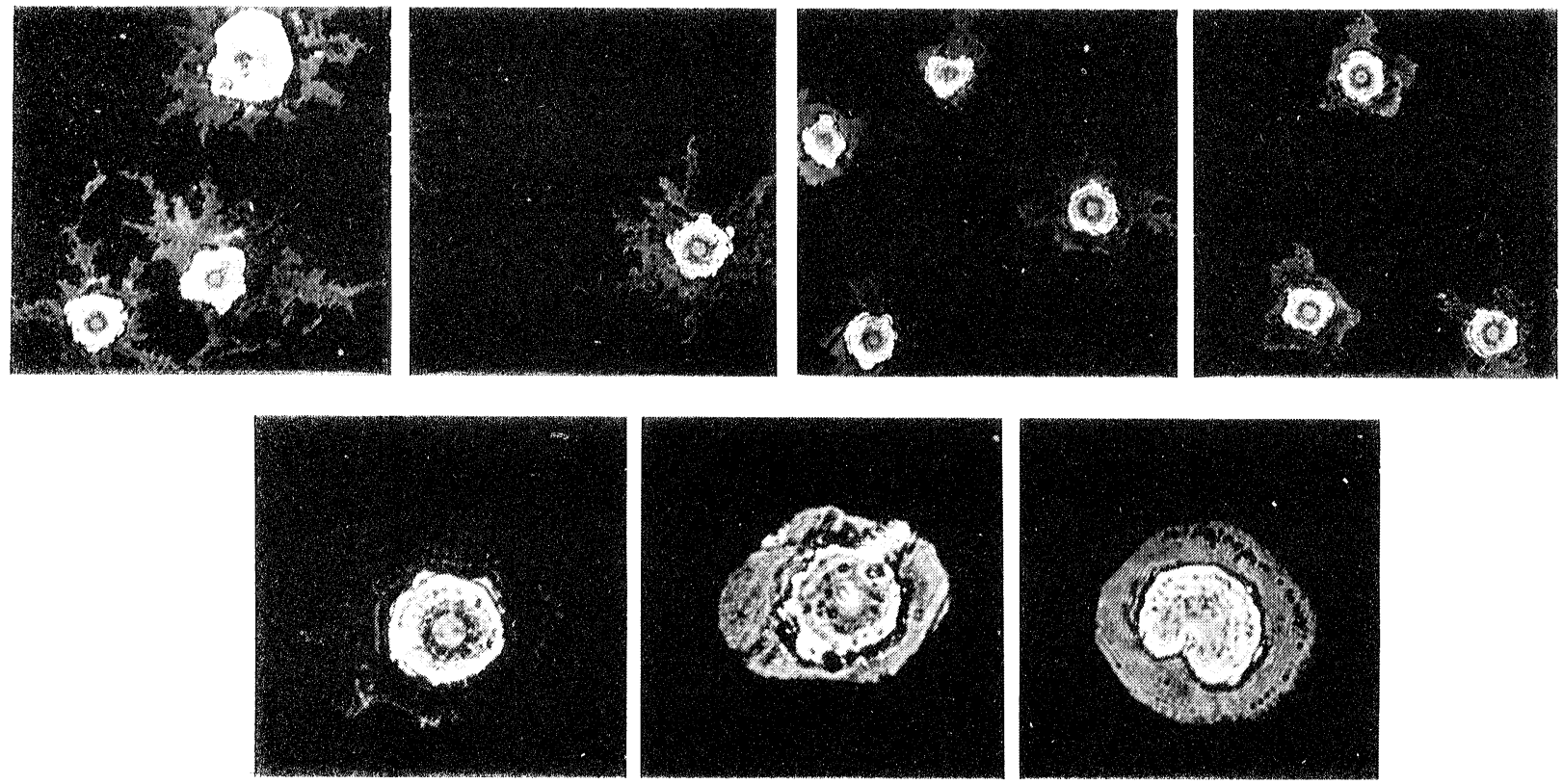

FIGURE 4 The dependence of the shape of the hole on electrode thickness. Film thickness $\mathrm{d}=2000 \AA$ and electrode thicknesses $\quad$ (a) $=990 \AA ; \quad$ (b) $=1200 \AA ; \quad$ (c) $=1900 \AA$; (d) $=2400 \AA ;(e)=2600 \AA ;(f)=3200 \AA$ and $(g)=3500 \AA$, respectively. [Magnification $\times 235$.] 
balance and the conditions of breakdown event ${ }^{1}$. It appears from these calculations that only a small part of the energy supplied is expended on the formation of a single hole breakdown. Energetic efficiency $\eta$ of such a process is low (column 10, Table 1) which may suggest that several self-healing breakdowns take place simultaneously. This may be connected with the existence of microscopically weak spots and other structural inhomogeneities in thin polymer films.

The dimensions and shape of the evaporated surface of the electrode are related to its thickness, the polymer film thickness being constant. Figure 4 shows a series of micrographs of breakdown channels for a polystyrene film $2000 \AA$ thick, provided with a gold electrode of linearly growing thickness. For the lowest thickness of the electrode equalling $990 \AA$ the evaporated area of the electrode is irregular, very ragged in shape. As the thickness of the electrode increases the shape of the hole becomes more and more regular and at electrode thickness of $3500 \AA$ the shape of the evaporated electrode is distinctly circular.

For all the examined samples a regular linear dependence has been found between the components of discharge energy $E$ and the mean breakdown channel surface in the polymer film $S_{p}$ and the mean surface of the evaporated electrode $S_{m}$. This dependence for a polystyrene film characterized by layer thickness $2500 \AA$ and upper electrode thickness $1500 \AA$ is shown in Figure 5. In this figure one can see the linear dependence of the hole surface $S_{m}$ relative to $\sqrt{ } E$. Figure 6 presents the $S_{m}$ vs $S_{p}$ dependence for the same polystyrene sample. It

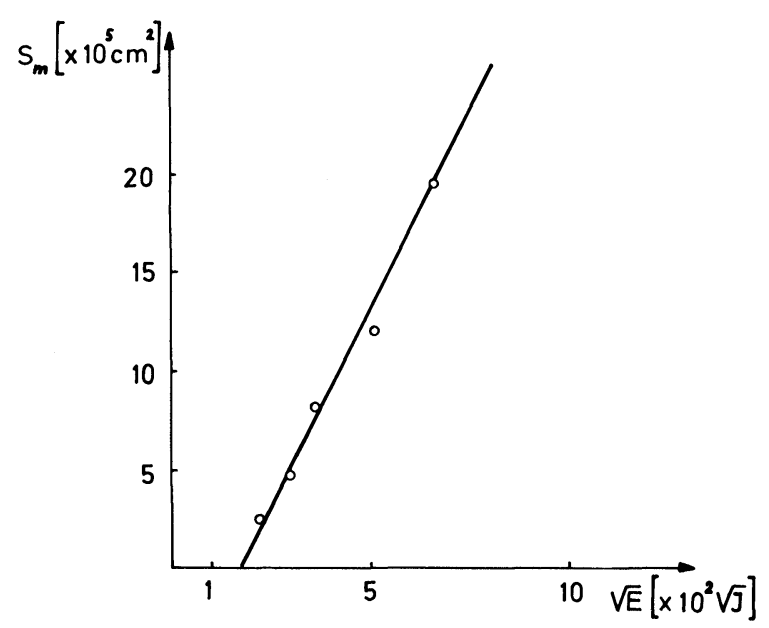

FIGURE 5 The dependence of the area of evaporated electrode on breakdown energy.

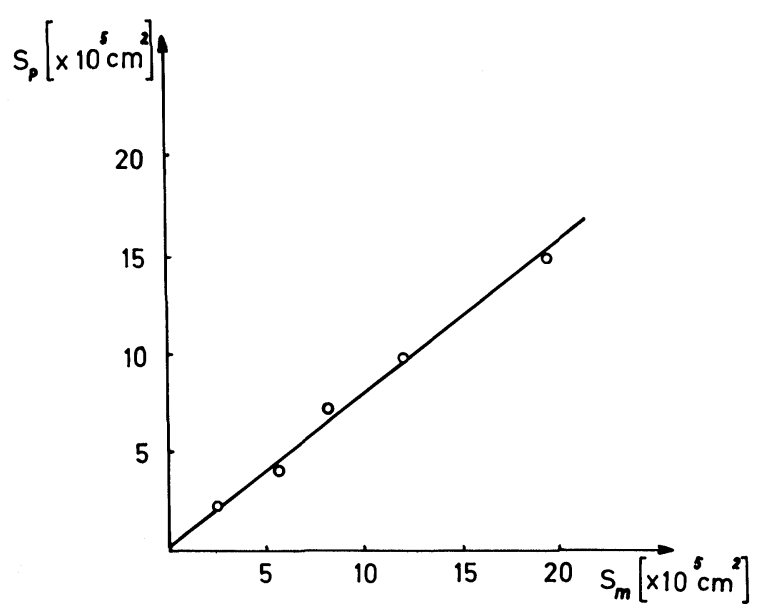

FIGURE 6 The relation between evaporated area of polymer and electrode. Polystyrene layer 2500 A thick.

appears from this plot that these values are directly proportional.

Flaws and inhomogeneities in the support have a marked effect on the weak spot formation. This can be seen in Figure 7 where breakdowns occur along the cracks in the support. Structural investigations of pure polybenzene films have also revealed the formation of large spherulite concentration along the crack in the support (Figure 3). This fact makes the interpretation of the nature of weak spots much more difficult, although it appears that in this case cracks rather than spherulite structures affect weak spot generation. In principle, spherulite structures should

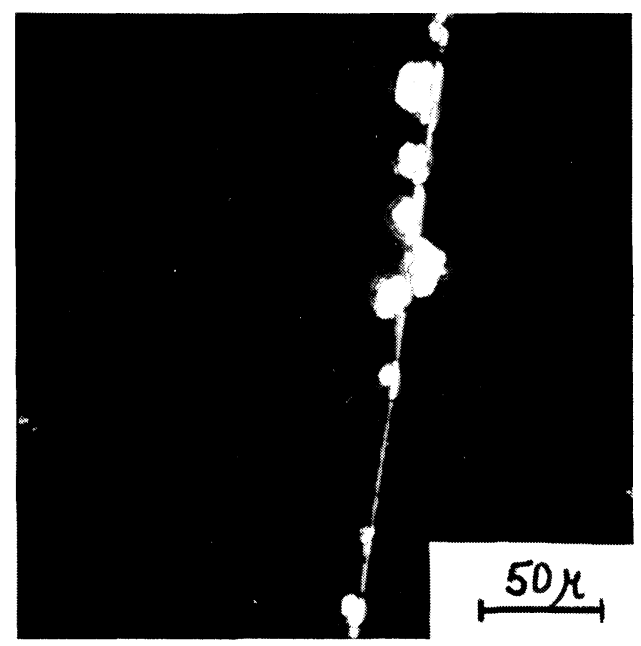

FIGURE 7 The effect of flaws in the support on weak spot generation in the film. 


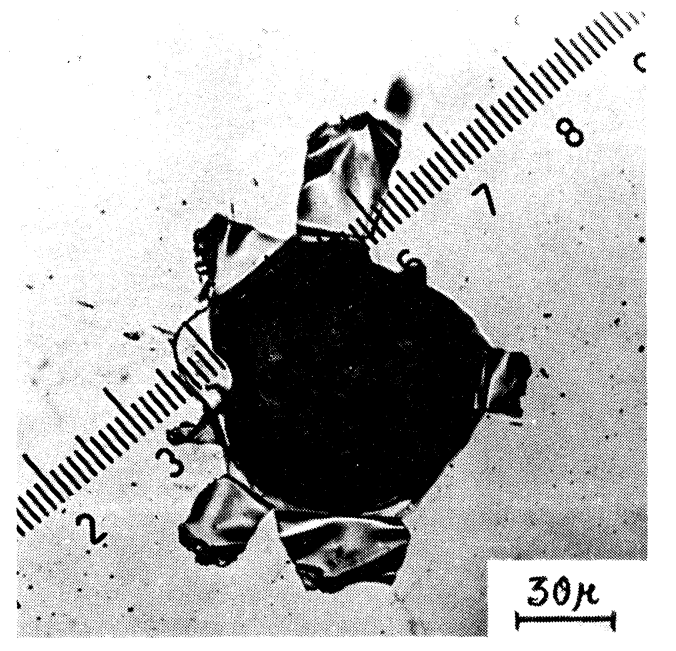

FIGURE 8 Breakdown channel in polystyrene $(d=2500 \AA)$. Optical microscope.

increase and not decrease the dielectric strength of the film ${ }^{9}$.

Typical micrographs of weak spots after breakdown for thin polystyrene films $2500 \AA$ thick, obtained by optical microscopy and examined in transmitted light, are presented in Figure 8. Micrographs shown in Figure 9 refer to identical polybenzene films. In both cases breakdown energy was $26 \times 10^{-4} \mathrm{~J}$. More details can be seen in electron micrographs. The interior of a crater in a polystyrene film $2500 \AA$ thick is presented in Figure 10. The mean dimensions of the structures that can be seen in

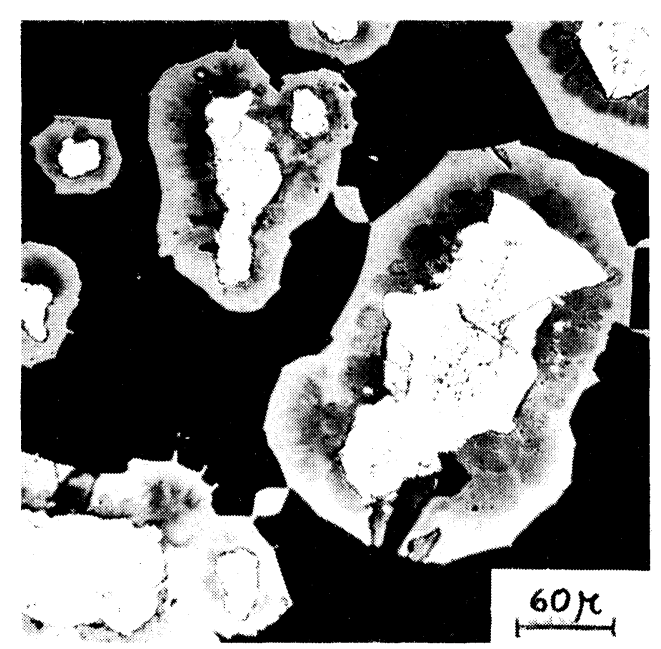

FIGURE 9 Breakdown channel in polybenzene $(d=2500 \AA)$. Optical microscope.

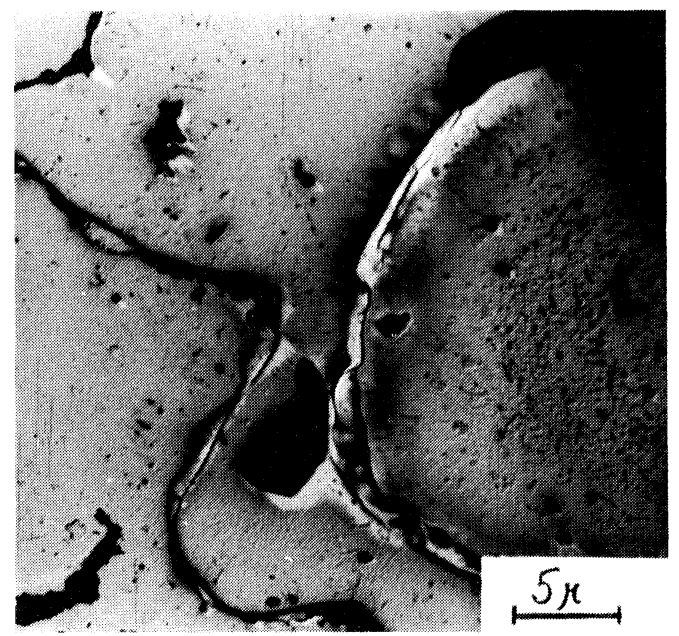

FIGURE 10 Electron micrograph of the interior of the channel in polystyrene $(d=2500 \AA)$.

the micrograph are of the order of $1100 \AA$ and their height is about $600 \AA$. The size of these structures is greater than the size of supermolecular structures observed on the surface of a freshly prepared film, where the mean dimensions of the nodules are $500 \AA$ and their height is $200 \AA$, for films three times as thick ${ }^{5,6}$.

Self-healing microbreakdown in polystyrene shaped like a deformed cone is demonstrated in Figure 11. The diameter at the base of the cone is $1.6 \mu \mathrm{m}$ and its $h_{\max }$ is $7500 \AA$. Distinct structures $450 \AA$ in diameter can be seen outside. Different

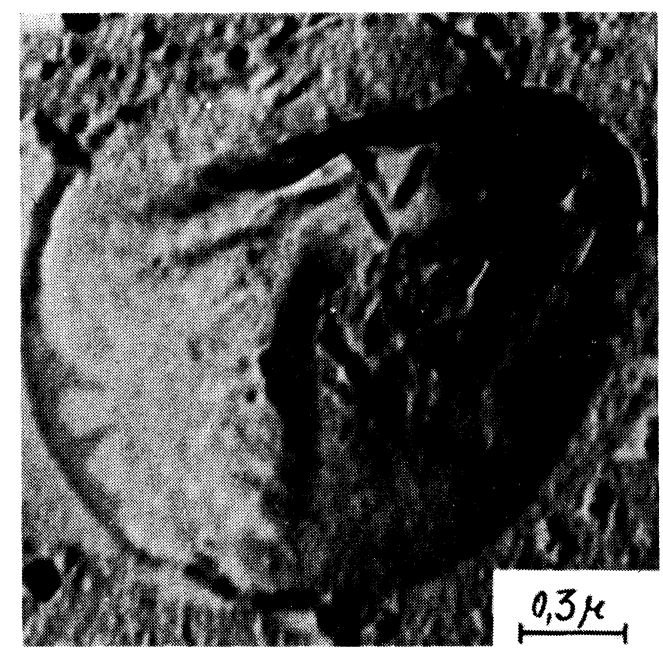

FIGURE 11 Electron micrograph of microbreakdown in polystyrene $(d=2500 \AA)$. 


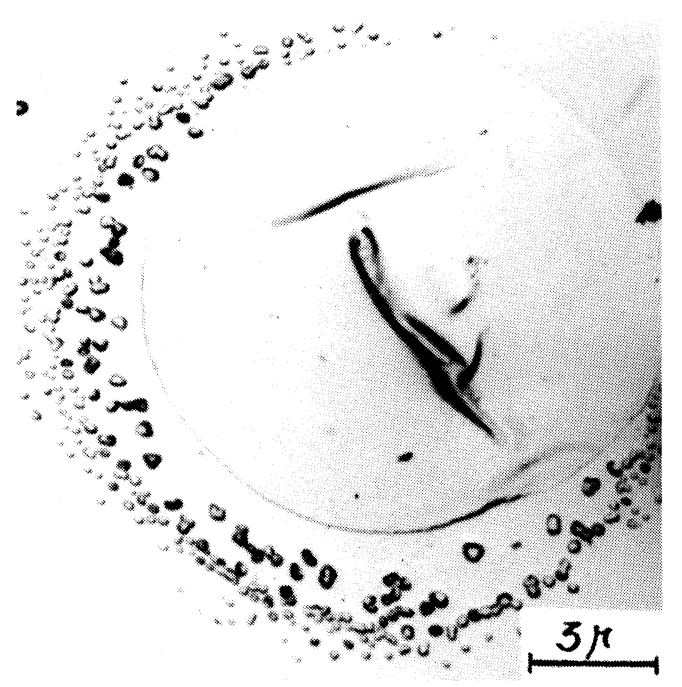

FIGURE 12 Electron micrograph of microbreakdown in polybenzene $(d=2500 \AA)$.

forms can be seen in polybenzene films. Figure 12 shows a microbreakdown shaped like a flat cone with distinct breakdown crater and polymer as well as electrode material ejected outside as a result of microexplosion which is evidenced by concentric arrangement of droplets. The breakdown diameter is $11,0 \mu \mathrm{m}$, the length of the crater being $5.3 \mu \mathrm{m}$. Figure 13 presenting the borderline area between the breakdown site and the undamaged film reveals a difference in structure inside and outside the crater. Distinctly visible elements on the right are micro-

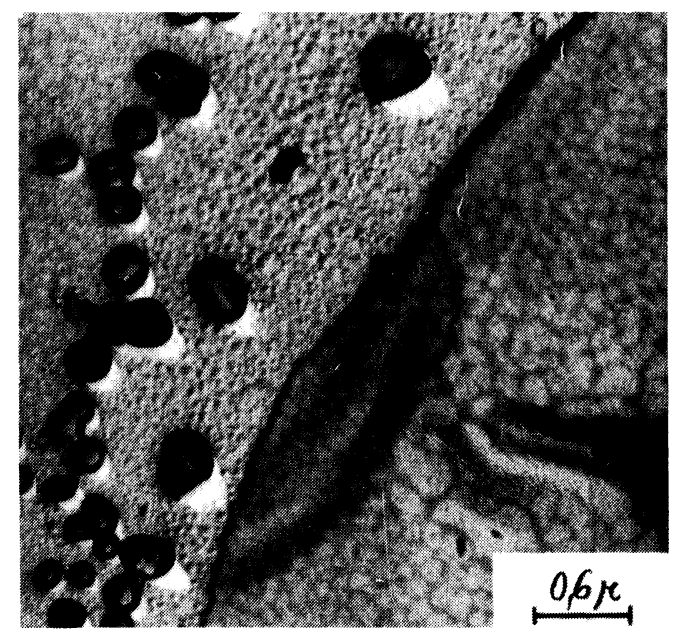

FIGURE 13 Borderline area between destroyed and undamaged film for the breakdown in Figure 12.

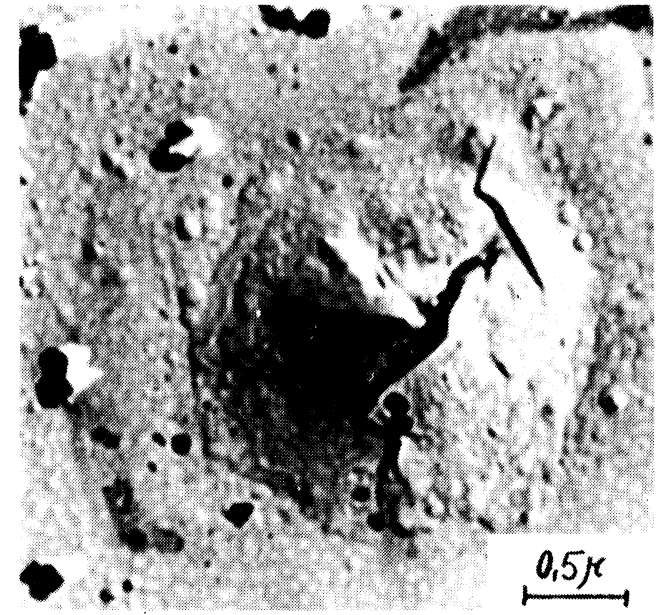

FIGURE 14 Typical breakdown crater for polybenzene $(d=2500 \AA)$. Crater's diameter $1.9 \mu \mathrm{m}$ and height $0.64 \mu \mathrm{m}$.

explosion products and their mean dimensions are contained within the range of $1700-2900 \AA$, while the structure of undamaged area is typical for polybenzene films, the dimensions of its elements being 300-400 $\AA$. Distinct breakdown craters are shown in Figures 14 and 15. Their dimensions are similar and equal $1.9 \mu \mathrm{m}$ and $2.2 \mu \mathrm{m}$ respectively. In Figure 14 one can see in addition a fold surrounding the cone proper $3 \mu \mathrm{m}$ in diameter. Distinct droplets scattered around the craters are the evidence of eruption brought about by thermal effects of the breakdown event. Their dimensions reach $1100 \AA$ and

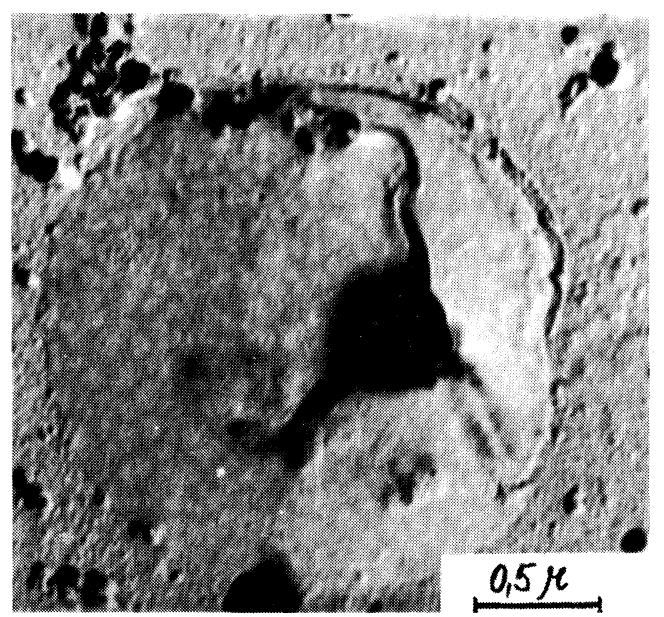

FIGURE 15 Typical breakdown crater for polybenzene. Crater's diameter $2.2 \mu \mathrm{m}$ and height $0.33 \mu \mathrm{m}$. 


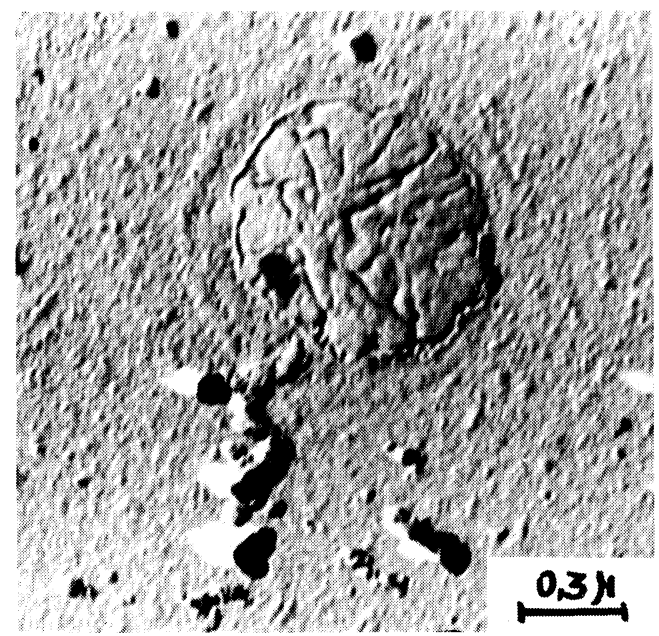

FIGURE 16 Breakdown crater in polybenzene with supermolecular structure.

their height is $500-550 \AA$. Figure 16 also shows a cone-like product but this time it has some structure. The fold on the left is similar to the one in Figure 14. The diameter of the cone at the base equals $9600 \AA$ and $h=2900 \AA$. Further structures can be seen in Figure 17. The distinctly superficial structure of a large microfragment is probably due to heat effects of breakdown and the follow-up phenomena. There is evidently no globule-like material ejected in the vicinity of the crater which may be due to formation of a different structure out of this material. Figure 18 leads to the same conclusion.

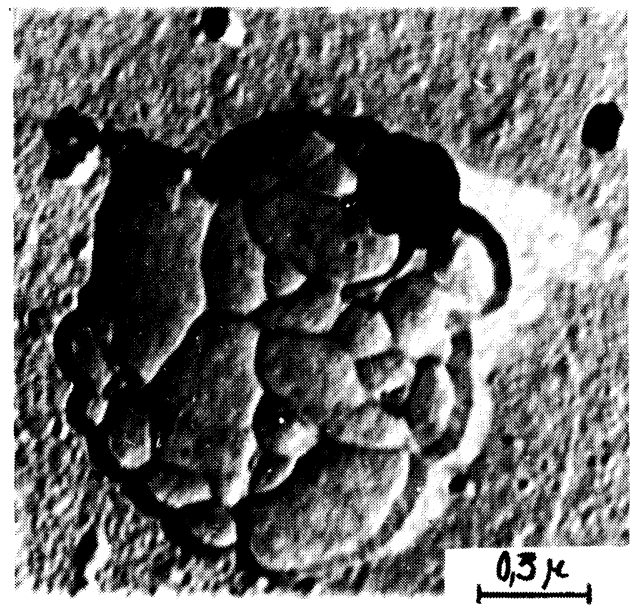

FIGURE 18 Atypical form of breakdown in polybenzene of $1.1 \mu \mathrm{m}$ in diameter and average height of $1500 \AA$.

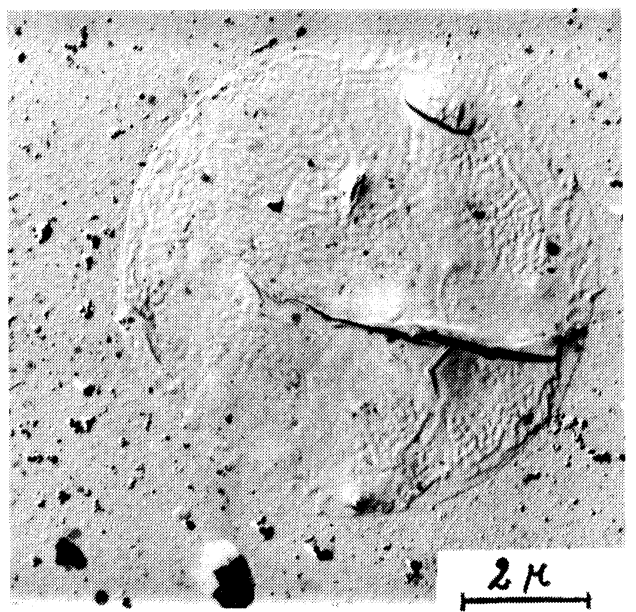

FIGURE 17 Atypical form of breakdown in polybenzene of $7.7 \mu \mathrm{m}$ in diameter. The dimensions of internal structures of the globules equal $1100 \AA$.

\section{DISCUSSION}

The analysis of the structures arising in breakdown sites leads to the following conclusions:

1) Within the range of breakdown energy level lower than $1 \times 10^{-5} \mathrm{~J}$ nondestructive breakdown take place.

2) For energy levels higher than $5 \times 10^{-3} \mathrm{~J}$ single hole self-healing breakdown with complete destruction of the inner morphological channel structure occurs.

3) Within the intermediate energy range $1 \cdot 10^{-5} \mathrm{~J}<\mathrm{E}<5 \times 10^{-3} \mathrm{~J}$ partial destruction of the breakdown channel takes place. Morphological structures found inside breakdown channels are similar to the ones occuring in a freshly prepared film. Electron micrographs reveal nodular-like structures (Figure 2). Due to overheating of the material deformed structures arise, resembling cones in shape (Figure 11). The evaporated polymer, and electrode material is deposited around the crater in the shape of droplets. In the cones there are fissures (Figures 12 and 14).

4) Formation of structures inside the breakdown channel (cones, local folds) may be due to branching of the current path at breakdown site, which is connected with the structural inhomogeneity of thin films.

5) Breakdowns start in the structurally most inhomogenuous areas and spread therefrom, involving wider areas of up to $1 \mu \mathrm{m}$ in diameter. A number of 
such microscopic areas make up a macroscopic destruction area a few microns in diameter. These structural inhomogeneities give rise to nonuniformity of current density and local overheating, which, in turn, may bring about local structural changes. Such structural microinhomogeneities in the film surface may be due to locally changing conditions of polymerization in the course of thin film preparation, among other factors. Both the analysis of the morphological structure of weak spots after breakdown and the analysis of electrical conductivity measurements of weak spots ${ }^{8}$ allow for the following conclusions concerning their nature to be drawn:

a) they may be the spots characterized by high electrical conductivity $\sigma$. These develop due to nonuniformity of discharge current density, or because of local overheating of the arising layer in the process of polymerization, which brings about a change in the $\mathrm{C} / \mathrm{H}$ ratio and higher values of $\sigma$ connected with it.

b) spots arising due to locally lower polymerization efficiency, brought about by changing rate of flow of fresh monomer into the support, by spots of locally different layer temperature, and related to this, by the reduced adsorption of the monomer on the surface of the newly forming polymer.

c) places connected with inhomogeneity of the support. These have been identified in the investigation of specially prepared films obtained on notched supports. In these cases weak spots are connected mainly with different thickness, which is evidenced by breakdowns occurring along the notches.

d) areas in which supermolecular structures occur giving rise to internal strains and local variations in thickness.

e) spots in which oligomers, capable of undergoing crystallization, arise. This radically changes the conditions of electron propagation.

f) places with monomer bubbles trapped in the film that have not undergone polymerization. Such places are characterized by reduced dielectric strength.

It follows from the foregoing remarks that in order to obtain films with minimum number of weak spots the following conditions must be satisfied:

a) high uniformity of current density in glow discharge,

b) uniform flow of monomer onto the surface of the polymer being formed, c) cooling of the support.

d) low polymerization rates so that maximum number of radicals can react with monomer vapours,

e) the support should be homogenous and free from flaws,

f) high frequency of discharge current.

The analysis and comparison of morphological structures of film surfaces and breakdown channels leads to yet another conclusion. The temperature in the center of the breakdown channel is considerably higher than the temperature at which thermal degradation of the polymer takes place. In the channel there also exist supermolecular structures, similar to those observed on the surface of a newly formed polymer $5,6,7$. This fact can be interpreted to mean that supermolecular structures on the surface of freshly formed film are also dependent on the existence of high local concentrations of degraded and highly reactive products.

Although the microscopic nature of weak spots is not yet fully known, it can be assumed that apart from inhomogeneities of structure it it also connected with the internal properties of such films. This is suggested by structural investigations of thin films in which crystalline structures have been found ${ }^{5,6}$, Similar studies carried out on polyethylene $e^{10,12}$ and polyethylene terephtalate ${ }^{11}$ films have clearly revealed a change in breakdown field depending on the crystallinity and molecular weight of the polymer. These facts, related to the structure of thin polymer films, their ability to undergo crystallization, the spatial inhomogeneity of the network extent and the change of all these quantities with ageing of the film, explain both the generation of weak spots and the change in dielectric strength in the function of film ageing.

\section{REFERENCES}

1. N. Klein, Advances in Electronic and Electron Physics, Ed. L. Marton, 26, 309 (1969).

2. P. P. Budenstein, P. J. Hayes, J. L. Smith, W. B. Smith, J. Vacuum Sci. Technol., 6, 289 (1969).

3. P. P. Budenstein, P. J. Hayes, J. Vacuum Sci. Technol., 6, 602 (1969).

4. S. L. Madorsky, Thermal Degradation of Organic Polymers, (John Wiley, New York 1964).

5. M. Kryszewski, "Formation of supermolecular structures in thin polymer layers" (Main Lecture in 8th Prague IUPAC Microsymposium 1971).

6. M. Kryszewski, J. Pure Appl. Chem., 31, 21 (1972).

7. D. Häfer, H. J. Tiller, K. Meyer, Plaste u. Kautschukk, 5, 354 (1972). 
8. S. Sapieha, Ph.D.Thesis, (Technicial University, Lódź 1972).

9. S. N. Kolesov, L. N. Chieraskov, Vysokomol.Sojedin., B 12, 266 (1970).
10. A. S. Silver, Wire a Wire Prod., 33, 102 (1958).

11. J. Artbauer, J. Griac, Kolloid-Z u. Z. Polymere., 205, 162 (1965).

12. R. A. Fava, Proc. Inst. Elec. Eng., 112, 819 (1965). 

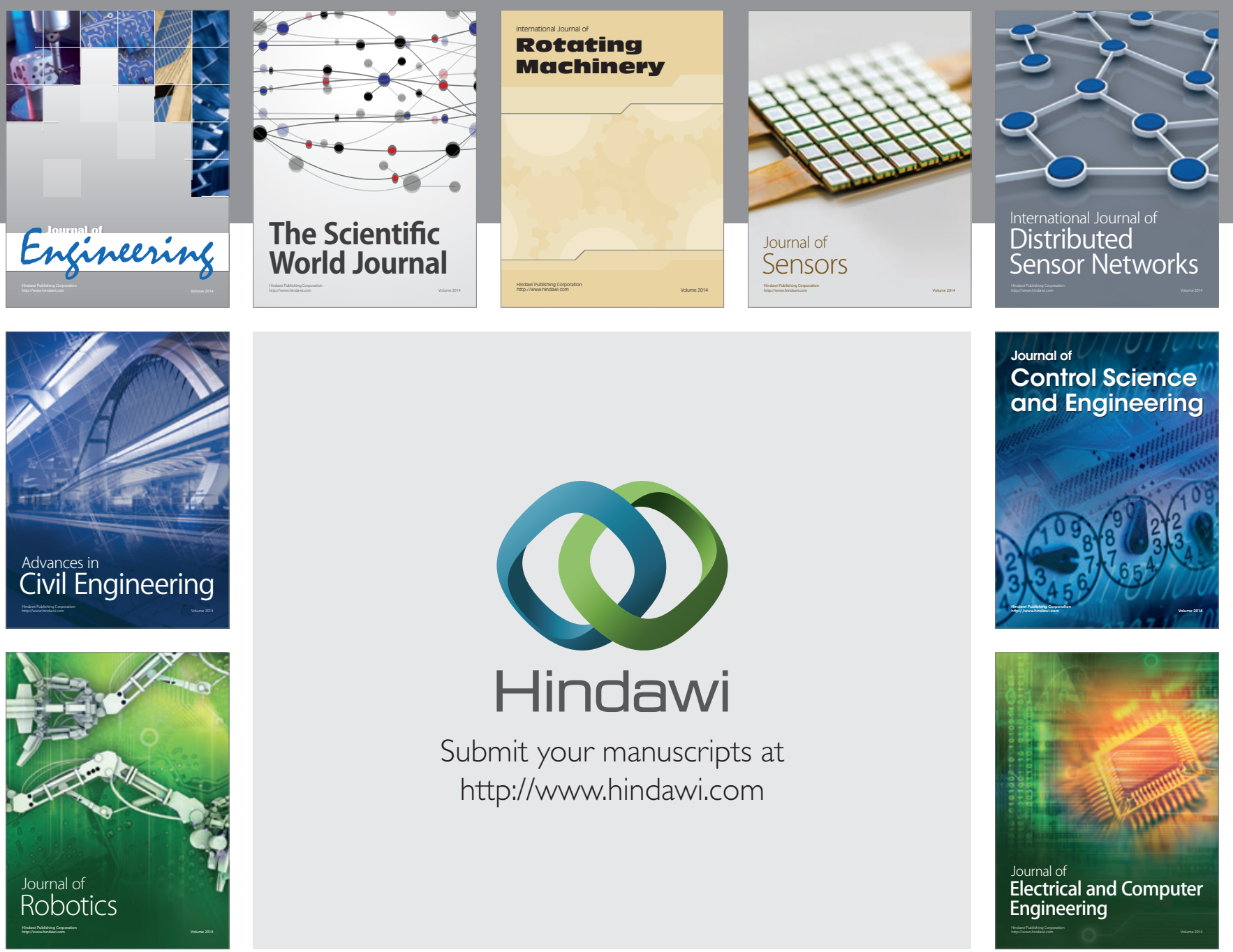

Submit your manuscripts at

http://www.hindawi.com
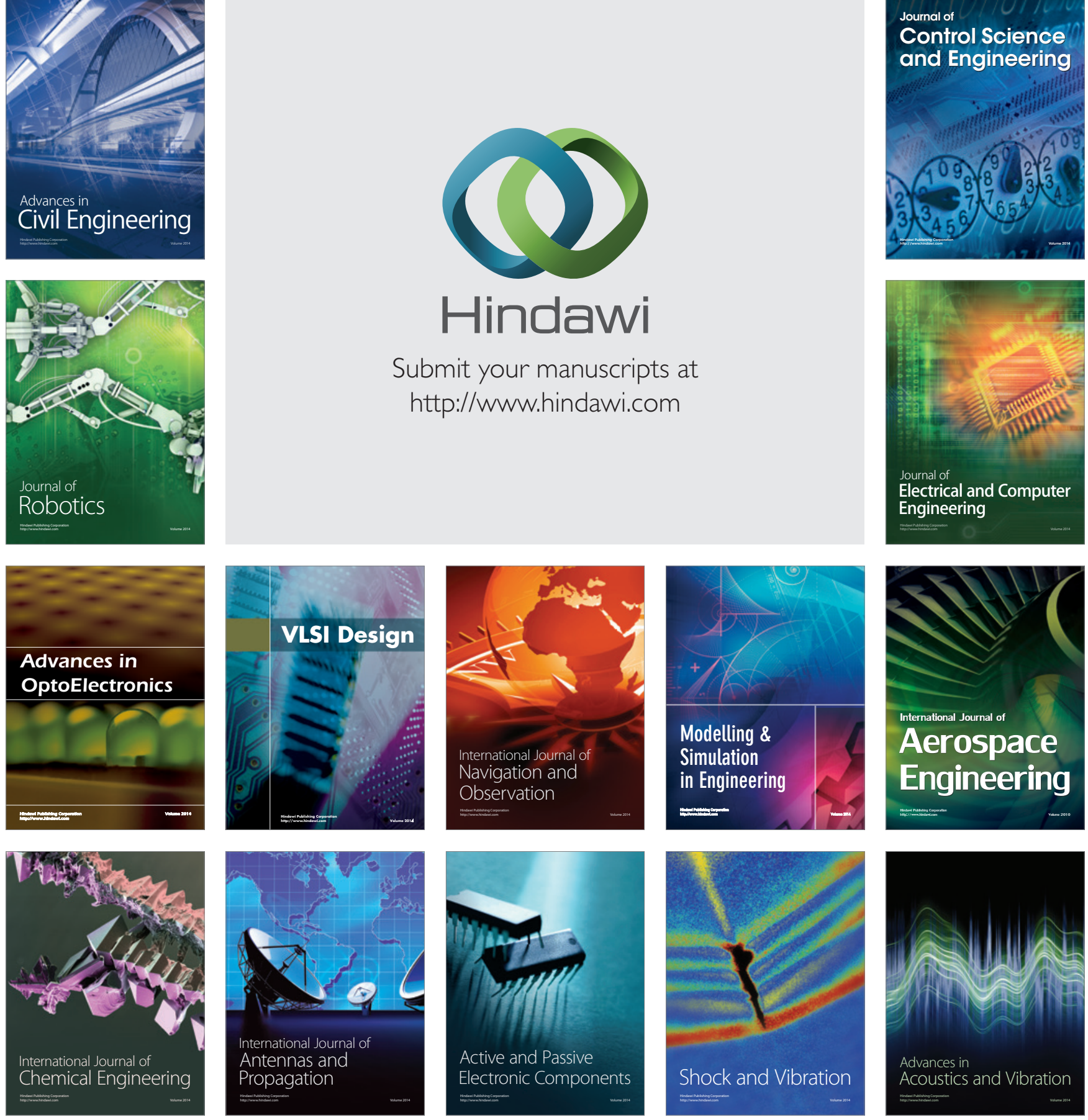